\title{
Un análisis de la unidad por analogía a partir de los modelos geométricos en el De Incessu Animalium y el De Motu Animalium de Aristóteles
}

\author{
An analysis of the unit by analogy from the geometric models in the $D e$ \\ Incessu Animalium and the De Motu Animalium
}

\author{
ÁNGEL AUGUSTO PASQUALE'
}

\begin{abstract}
Resumen: En este trabajo exploro ciertas modelizaciones en la obra zoológica de Aristóteles. En especial, me interesa el tipo de esquemas teóricos en los cuales Aristóteles no pretende precisión anatómica ni fisiológica porque hacen referencia a más de un género de animales o sus partes, o de actividades. Analizo por un lado el esquema triaxial de las facultades de los animales, en el cual las dimensiones quedan definidas no por su posición sino por una función, presente en el De Incessu Animalium 4. Por otro lado, tengo en cuenta el diagrama del De Motu Animalium 9, en el cual Aristóteles concluye que el medio material del animal, el corazón, no puede originar movimientos simultáneos, por lo que tiene que haber algo que mueva a los dos sin ser movido. Considero que este modelo distingue también sus elementos no por sus posiciones sino por sus funciones. Los términos geométricos asignados en cada caso dan cuenta de determinadas funciones de los animales, presentando un valor doble de la analogía: entre el dominio animal y el geométrico, y otorgando la generalidad necesaria para captar lo semejante entre géneros distintos entre el mismo dominio animal.
\end{abstract}

Palabras clave: Aristóteles; biología; analogía; modelos; geometría.

\begin{abstract}
In this article I explore certain modeling in Aristotle's zoological work. In particular, I am interested in the type of theoretical schemes in which Aristotle does not claim anatomical or physiological precision because they refer to more than one genus of animals or their parts or activities. On the one hand, I analyze the triaxial scheme of the faculties of animals in the De Incessu Animalium 4, in which the dimensions are defined not by their position but by a function. On the other hand, I examine the diagram of the De Motu Animalium 9, in which Aristotle concludes that the material middle of the animal, the heart, cannot originate simultaneous movements, so there must be something that moves without being moved. I believe that this model also distinguishes its elements not by their positions but their functions. The geometric terms assigned in each case account for certain functions of animals, presenting a double value of the analogy: between the animal domain and the geometric one, and granting the necessary generality to capture the similarity between different genus within the animal domain itself.
\end{abstract}

Keywords: Aristotle; biology; analogy; models; geometry.

Cómo citar: Pasquale, A.A. (2020). Un análisis de la unidad por analogía a partir de los modelos geométricos en el $D e$ Incessu Animalium y el De Motu Animalium de Aristóteles. Cuadernos Filosóficos 17. DOI: https://doi.org/10.35305/cf2.vil 7.84

Publicado bajo licencia Creative Commons Atribución-SinDerivadas 4.0 Internacional [CC BY-ND 4.0] (c) (1) $\odot$ 


\section{ÁNGEL AUGUSTO PASQUALE Un análisis de la unidad por analogía a partir de los modelos geométricos}

Fecha de aprobación: 21/07/202I

\section{Introducción}

En 1978 Martha Nussbaum inaugura ciertas líneas con respecto al papel de la analogía en la investigación de Aristóteles sobre el movimiento de los animales que fueron por muchos años dominantes (Nussbaum, 1985). De hecho, los papers más recientes acerca de la teoría aristotélica del movimiento de los animales siguen discutiendo con lo expuesto en sus célebres ensayos ${ }^{2}$. Nussbaum parte de una separación relativamente tajante entre un plano cosmológico y uno biológico, a partir de la cual las referencias al movimiento del cielo en la investigación del movimiento animal fundan una analogía, en el sentido de que los principios del primero se aplican al segundo, pudiendo así ser probados (Nussbaum, 1985, Pp. 120, 140-42). Por otra parte, respecto a las analogías con la geometría presentes también en estas discusiones, ella afirma que son de poca importancia para las argumentaciones ${ }^{3}$. En este trabajo intento dar cuenta de dos pasajes de la investigación zoológica del movimiento de los animales en los que se pone en juego el recurso analógico: por un lado, del esquema tridimensional de las facultades del alma que Aristóteles presenta en el capítulo 4 del De Incessu Animalium, en el que se definen las dimensiones no por sus posiciones con respecto al lugar del cielo y de la tierra sino por su función, y por otro, del argumento de la localización del alma motriz en el capítulo 9 del De Motu Animalium, momento de la argumentación en el que se ofrece un diagrama para mostrar por qué debe haber otra cosa que mueva a los principios en caso de movimientos simultáneos. Mi aproximación a ambos argumentos dista de la línea de Nussbaum puesto que procuro examinar el recurso analógico respetando la naturaleza de la analogía tal como Aristóteles la concibe, o al menos las pistas que brinda, siguiendo investigaciones más recientes que se comprometen con esa empresa ${ }^{4}$. Considero que la analogía es la manera de poder tratar el movimiento de los animales, actividad tan desemejante entre los diferentes

2 Véase, por ejemplo, Falcon (20I7) que, aunque es un artículo reciente, el autor se esfuerza por distanciarse de la presunta interdisciplinariedad del tratado De Motu Animalium propuesta por Nussbaum.

3 De la reciente lectura crítica noto dos grandes maneras muy distintas de interpretar los modelos que representan los movimientos de los animales en Aristóteles. Por un lado, la lectura impulsada por Nussbaum, a partir de la cual los modelos geométricos son considerados de poca relevancia al argumento puesto que se pueden explicar las situaciones sin aquellos e incluso no logran mostrar los aspectos más significativos de la imagen fisiológica. En esta lectura, el modelo geométrico, por ejemplo en MA I, se inserta en la argumentación por la que Aristóteles estaría aplicando por analogía principios ya establecidos en la cosmología ahora al movimiento de los animales (1985, pp. 277, 28I, 283-4). Por otro lado, encontramos la línea de la cual Thómas Bénatouil es un exponente con su investigación sobre el MA (2004), y su interpretación queda detallada en la siguiente nota. 


\section{ÁNGEL AUGUSTO PASQUALE Un análisis de la unidad por analogía a partir de los modelos geométricos}

géneros que de otro modo no podría ser considerada una sola sino por su función. Sin embargo, los pasajes elegidos presentan otra característica: las actividades y partes de los animales son comparadas con modelos geométricos, lo que establece un nivel de abstracción mayor para explicar los casos de la zoología en general.

\section{El modelo de De Incessu Animalium 4}

En el capítulo 4 del De Incessu, Aristóteles presenta una división de los seres vivos a partir

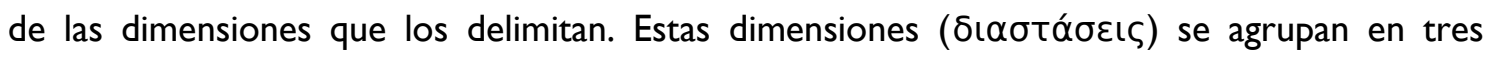
pares de opuestos ( $\alpha \dot{\nu} \tau(\kappa \varepsilon i ́ \mu \varepsilon v \alpha$ ), lo alto y lo bajo, lo delantero y lo trasero, y la izquierda y la derecha. En De Caelo I, I define a los cuerpos como magnitudes continuas que se extienden en tres dimensiones, a diferencia de las líneas y las superficies (268a6-9). Distanciándose de este plano físico-geométrico, Aristóteles advierte en De Incessu que los pares de opuestos de

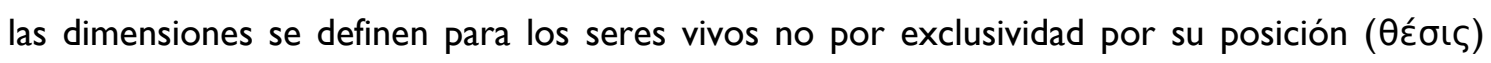
respecto de la tierra y el cielo, sino por su función (દ̌pyov), y que, entre los pares, lo alto, lo delantero y la derecha son principios, y así funda un nuevo plano ahora biológico-funcional de las dimensiones ${ }^{5}$. La dimensionalidad físico-geométrica y cósmica no coinciden sin más, sino

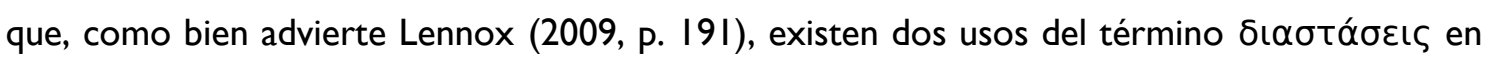
De Caelo, que no son meros homónimos, sino que el plano físico-geométrico y el cosmológico se conectan al afirmar que los atributos direccionales son fuentes de las tres dimensiones corporales. Sea como fuere, Aristóteles construye una axialidad de las funciones animales, y

4 Bénatouil (2004, pp. 93-5) afirma que la investigación del MA debe explicar el movimiento de los animales por sí mismos dentro de su campo disciplinar, la zoología -aunque sea lícito traer a colación principios generales de la naturaleza ya establecidos. De esta manera el modelo geométrico cobra relevancia porque Aristóteles pretende dar cuenta la causa común del movimiento de los animales a través de recursos del propio campo disciplinar, y en este caso, de analogías. Y a su vez esta lectura entiende la relevancia de que el modelo aparezca nuevamente en los capítulos sucesivos para indicar el lugar del alma en el medio del animal, la relación instrumental que aquélla establece con el pneûma y la manera en que los estímulos se dirigen necesariamente al área central, y en todas estas argumentaciones Aristóteles se sirve de las funciones establecidas para los elementos involucrados en la flexión de la extremidad. De esta manera, el término desconocido de la comparación en el prólogo pasa a ser el término conocido en otros pasajes del tratado, y por tanto el modelo geométrico tal como es presentado en el primer capítulo otorga un marco de estructura y cohesión para la investigación desarrollada (Bénatouil, 2004, pp. 105-108).

5 La terminología de "espacio biológico-funcional" es de Bartolomé \& Marcos (2010, n. 613). Frente a este espacio de coordenadas, Marcos opone uno "físico y cosmológico". Mi interpretación es distinta puesto que considero que el recurso geométrico hace posible la comparación de los dos espacios -uno supralunar y otro superlunar, podríamos decir-, o quizá porque el término "físico-cosmológico" podría tratarse en todo caso de una redundancia. 


\section{ÁNGEL AUGUSTO PASQUALE Un análisis de la unidad por analogía a partir de los modelos geométricos}

aunque haciendo referencia a la argumentación del $D e$ Caelo, el modelo es justificado exclusivamente por la biología.

Reproduzco el pasaje del De Incessu que, aunque extenso, permite entender mejor cómo se desarrolla la argumentación:

Dado que las dimensiones con las que los seres vivos están delimitados por naturaleza son seis, lo alto y lo bajo, lo delantero y lo trasero, y, además, lo diestro y lo siniestro, todos los seres vivos poseen la parte de arriba y la de abajo. De hecho, no sólo en los animales existe lo alto y lo bajo, sino también en las plantas. $Y$ se diferencian por su función y no solamente por su colocación con respecto a la tierra y al cielo. $Y$, efectivamente, donde se produce la distribución del alimento y el crecimiento para cada uno es en lo alto; en cambio, donde este alimento llega a lo último es en lo bajo. Lo uno es un principio, lo otro un final; y el principio es lo alto. Sin embargo, podría parecer más propio de las plantas que fuera lo bajo, pues lo alto y lo bajo no tienen la misma posición en ellas y en los animales. Respecto al todo, la disposición no es igual, pero hay similitud respecto a la función. Efectivamente, las raíces son para las plantas la parte alta, pues desde allí se distribuye el alimento para los vegetales y lo toman a través de éstas, como los animales con la boca.

Todos los seres que no sólo viven, sino que también son animales, tienen parte delantera y trasera. De hecho, todos ellos tienen sensación y con respecto a ésta se delimitan la parte de atrás y la de delante: en efecto, la parte en la que surge la sensación y desde donde se extiende para cada uno de los animales es la parte delantera, mientras que la parte opuesta es la trasera.

Y en aquellos animales que no sólo tienen sensación, sino que pueden desplazarse por sí mismos, se distinguen, además de las partes citadas, la izquierda y la derecha, definidas cada una de ellas, como las anteriormente dichas, por una función y no por su posición, pues la parte del cuerpo donde reside por naturaleza el principio del desplazamiento es para cualquier animal la derecha, y la parte contraria y por naturaleza servidora de ésta, es la izquierda. (Aristóteles, De incessu, 705b30-706a256)

Este esquema resulta naturalmente en una exposición de la derecha como principio de movimiento de todos los animales, que Aristóteles prueba a partir de varios casos, pero tiene otros usos y ventajas en el contexto de la obra. En el capítulo 5, en un primer momento, se realiza una división de los animales según si tienen diferenciado lo alto y lo delantero: si los tienen diferenciados, son bípedos, como los seres humanos y los pájaros, de los cuales dos de sus cuatro puntos de apoyo no están en contacto con el suelo; si lo alto y lo delantero no se

6 Todas las citas del $I A$ y del MA siguen la traducción de Almudena Alonso disponible en Jiménez SánchezEscariche \& Alonso Miguel (2000). 


\section{ÁNGEL AUGUSTO PASQUALE Un análisis de la unidad por analogía a partir de los modelos geométricos}

diferencian, son cuadrúpedos, polípodos y ápodos (706a26-b3). En un segundo momento, caracteriza la orientación o postura de estos según el lugar de su alto funcional. Mientras que las plantas están orientadas hacia abajo, los cuadrúpedos, los polípodos y los ápodos lo están hacia el medio, y los bípedos hacia lo alto del universo, porque están derechos, y los que más erguidos están son los humanos, los más conformes a la naturaleza (706b3-10). Aquí parecen relacionarse los planos biológico-funcional y físico-geométrico, a los que Aristóteles presentó y diferenció anteriormente. Sin embargo, una vez más, Aristóteles destaca la prioridad y autonomía del punto de vista funcional. Por más que la contrastación de los dos ejes dimensionales a veces revele correspondencia, la definición de polaridades con respecto a la orientación del universo no es relevante para la definición de polaridades desde el punto de vista funcional. Según Carbone (2005, pp. 235-36), la serie de polaridades cosmológica es un punto de referencia necesario para enmarcar el tratamiento comparativo de la organización de los animales según las polaridades funcionales y establecer una escala orientada desde una condición de excelencia. Es en este sentido que puede decirse que los seres humanos son los más excelentes por naturaleza porque la posición de su principio coincide con la del universo. Como afirma Lennox (2009, p. 20I) lo que hace a los humanos especiales no es solo que sus principios de movimiento están en esos lugares, sino que hay un recordatorio de que el orden más natural es el alineado con la direccionalidad cósmica ${ }^{7}$.

En los argumentos por analogía se da un pasaje de la atribución del predicado del foro -"la proposición $A$ es verdadera en el caso $C l$ ", el término de la comparación más conocido- al tema - “ $A$ es verdadera en el caso $C 2$ ", el objetivo de la comparación. Este proceso de predicación se interrelaciona con un proceso de abstracción: lo propio de la analogía es abstraer una característica común a varias entidades diferentes en género. Esta similitud entre los términos analogados debe fundarse en una característica que no corresponda al género definicional -puesto que no coinciden en género-, abstrayendo así un género funcional o pragmático (Macagno, 2017, pp. 470-7I). Según Alfredo Marcos (1996, p. 55),

El mostrar que dos entidades son genéricamente iguales en algún aspecto nos sirve para trasladar lo que sabemos acerca de una, la que nos es más familiar bajo ese determinado aspecto, a la otra. El desplazamiento nos permite comprender rasgos de una entidad en términos de la otra que tenemos experiencia.

7 Como en $D C 2.2$ las coordenadas del cielo se establecen a partir de la funcionalidad biológica, se puede decir que las argumentaciones del $I A$ y del $D C$ se suponen mutuamente. De hecho, como queda claro por la caracterización de excelencia de los humanos, del arriba y del abajo Aristóteles tiene una explicación enteramente no biológica, y esta es usada regularmente en contextos biológicos. Cfr. Lennox (2009, p. 203). 


\section{ÁNGEL AUGUSTO PASQUALE Un análisis de la unidad por analogía a partir de los modelos geométricos}

Geoffrey Lloyd identifica un uso zoológico de la analogía en Aristóteles. Mientras que las diferencias entre distintas especies del mismo género se tratan de "el más y el menos", algunas diferencias de las partes de los animales y sus actividades son las mismas solo por analogía, como ocurre entre animales de diferentes géneros (Lloyd, 1996, Pp. 140-4I).

Por otra parte, considero al modelo un tipo especial de analogía. Siguiendo al Diccionario de la Lengua Española decimos que es una representación o esquema teórico, generalmente en forma matemática, de un sistema o realidad más compleja, que se elabora para facilitar su comprensión y el estudio de su comportamiento ${ }^{8}$. Es decir, implica un flujo de sentido de lo más familiar a lo más extraño. En tanto se facilita la comprensión de un dominio a través de otro, su carácter explicativo y probatorio es limitado. Es por esto que su utilización se reserva a contextos heurísticos. En la filosofía natural aristotélica contamos con abundantes modelizaciones geométricas y mecánicas, no solo en biología sino en meteorología, astronomía y física general'.

En el pasaje del $I A$ en cuestión, Aristóteles realiza una buena utilización de un modelo, ya que se esfuerza en distinguir el modelo y lo que trata de elucidar. Mientras que en el modelo las dimensiones o polaridades son posiciones fijas respecto del universo, para los animales las dimensiones se definen por su función. Owen $(1986$, p. 318) sugiere que en tanto la interpretación de las abstracciones puede ser suministrada y las diferencias advertidas, los modelos son así herramientas indispensables, y no descripciones de un mundo superior, platónico. El IA llega a la división o clasificación de los animales en géneros no esenciales, una división de hecho distinta a la propuesta en $P A$, a partir del recurso analógico en el propio plano biológico-funcional: a partir de la referencia a los principios, primero se distinguen los animales que se mueven de los que no, y luego, por la posición de dichos principios se divide a los animales según sus puntos de apoyo y la orientación de sus cuerpos.

\section{El modelo del De Motu Animalium 9}

En el caso del $M A$, el autor enseña que el lugar del alma motriz es una consecuencia necesaria de la simultaneidad de los movimientos contrarios. El argumento del capítulo 9 tiene dos momentos. En un primer momento (702b l2-25), Aristóteles afirma que dado movimientos contrarios desde la derecha y desde la izquierda que se dan al mismo tiempo, no ocurre que

8 Versión electrónica de la edición 23a disponible en https://dle.rae.es/.

9 Véase Owen (1986), en donde se analizan diversas modelizaciones en los mencionados contextos disciplinares. 
una permanezca inmóvil y la otra se mueva, y viceversa, por lo que es necesario que el principio del alma motriz esté en el medio, ya que es un límite de ambos extremos. Y lo mismo ocurre para los movimientos que proceden de arriba y de abajo.

El segundo momento (702b25-703a3) supone el primero, pero la argumentación comienza nuevamente. Por más de que la parte del medio del cuerpo sea una sola potencialmente, en acto debe ser múltiple, porque cuando un miembro se mueve a partir del principio, otro permanece quieto. Para ilustrar esto Aristóteles nos ofrece un diagrama simple de tres puntos (que acompaña ocasionalmente algunas ediciones del texto ${ }^{10}$ ) y afirma:

\begin{abstract}
Me refiero, por ejemplo, a que en la figura $A B C, B$ es movido y $A$ mueve. Pero, ciertamente, es necesario que algo quede, si es que una parte debe ser movida y otra debe mover. Por tanto, aunque $\mathrm{A}$ es en potencia uno, en acto será dos, de modo que, necesariamente, no es un punto sino una magnitud. Sin embargo, sin duda, es posible que $C$ sea movido al mismo tiempo que $B$, de forma que es obligado que los dos principios que están en A muevan al ser movidos. Así, pues, tiene que haber algo fuera de estos principios que mueva sin ser movido. Efectivamente, las extremidades y los principios que están en $\mathrm{A}$ se apoyarían unos en otros al moverse, como quienes movieran las piernas apoyando las espaldas una contra otra. No obstante, es necesario que haya algo que mueva a los dos. $Y$ esto es el alma, que es algo distinto de la tal magnitud, pero que está en ella. (Aristóteles, De Motu Animalium, 702b25-703a I0)
\end{abstract}

En el capítulo posterior Aristóteles hace explícito que el medio del animal se trata en algunos animales del corazón y en otros de su análogo, y en ellos se encuentra también el pneûma, el soplo innato que hace de instrumento del alma para el movimiento (703a l4-16).

Coincido con Thomas Bénatouil (2004, Pp. 96-98) en que el modelo geométrico permite identificar el movimiento adaptado a la estructura de un objeto, pero ignora las condiciones concretas de este movimiento y no trasluce el proceso que produce el movimiento". Considero como él que los diagramas presentes a lo largo del MA replican el modelo del movimiento de las extremidades presentado en el capítulo I. Por más que, como Aristóteles mismo reconoce, la geometría no tiene en cuenta el funcionamiento específico de la

10

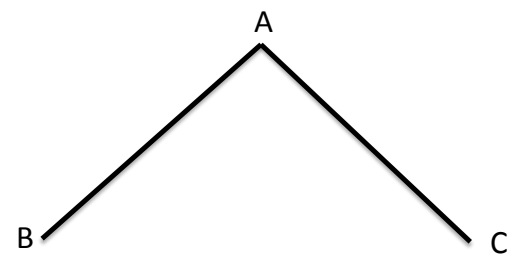

Cuadernos Filosóficos 17 (2020) 


\section{ÁNGEL AUGUSTO PASQUALE Un análisis de la unidad por analogía a partir de los modelos geométricos}

articulación, en este modelo se ofrece un diagrama del movimiento de las extremidades, que consiste en que la articulación puede dividirse en dos partes para permitir el movimiento de la extremidad y al mismo tiempo darle el soporte estable necesario para moverse. El modelo geométrico ofrece una representación abstracta de la posición inicial y de la posición final del objeto movido, pero no brinda solo una silueta exterior, sino que logra capturar la causa formal del movimiento, a la vez que es ciego a las causas materiales y motrices. En este sentido, la geometría no propone exclusivamente pasar de las analogías a la física; la geometría misma sería en cierta medida una explicación del movimiento. Sin embargo, la causa formal, considerada superior a las otras, tiene un rol menor frente a la causa material y al principio del movimiento para explicar la producción de un movimiento. Por esto el modelo geométrico no es suficiente para la investigación de la naturaleza.

Aristóteles distingue bien los planos geométrico y biológico en el argumento del capítulo 9. Entiende que el modelo trata como puntos lo que en realidad son magnitudes; incluso entiende que para las líneas el movimiento es una ficción ${ }^{12}$. Sin embargo, el mérito de la comparación radica en que se muestra que el punto $A$ puede ser considerado a la vez uno y doble, por tomar parte tanto del radio BA como del radio AC. Que sea doble es requisito para que haya movimiento, ya que mientras que uno se mueve, el otro debe permanecer quieto como punto de apoyo. En caso de movimientos simultáneos, los puntos se apoyarían mutuamente, y de ahí se sigue necesariamente la existencia de otra cosa inmóvil e inmaterial en el principio. La descripción geométrica resulta tan abstracta como para permitir su aplicación a una generalidad de animales y sus partes sumamente desemejantes. De esta manera se hace explícita la función, aquello en lo que sí se asemejan, tanto los animales que cuentan con

II Una posición distinta encontramos en De Groot (2008, p. 65). Ella afirma que estos modelos deben llamarse directamente modelos mecánicos, puesto que no son estrictamente matemáticos. Como la matemática se abstrae de la materia y el movimiento, Aristóteles busca modelos en otras ciencias matemáticas -mixtas, agregamos- que en sus principios incluyan al movimiento, como la astronomía y la mecánica. Para ella así son los casos de los modelos del MA: los puntos y líneas importan el "principio del radio móvil"-en su primitiva variante del movimiento de las circunferencias concéntricas- para mostrar la amplificación del efecto como un aspecto unificador del movimiento de los animales.

12 MA 1,698a24-26. Es interesante contrastar las dos nociones de "punto" que Aristóteles emplea en este tratado.

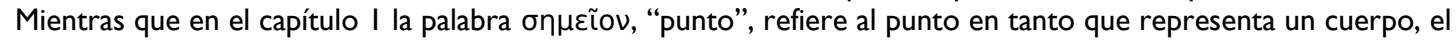

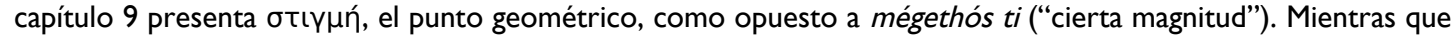
antes reconocía la ficcionalidad del punto en cuanto representaba magnitudes, ahora nos dice que el punto geométrico no se comporta de ningún modo como una magnitud. A este respecto, son relevantes las conclusiones de Vita (1982, p. 105): "Aristotele adopera ariy/irj quando vuole indicare il punto elemento di una linea, o di una figura in genere, elemento cioè appartenente all'insieme infinito di punti che caratterizza una figura geometrica, adopera invece atj/xelov quando vuole riferirsi al punto che appare nelle costruzioni geometriche, nelle dimostrazioni delle proposizioni o nelle interpretazioni matematiche dei fenomeni fisici. II primo termine appare quindi pertinente alla geometria nelle sue connessioni con la filosofia, nelle questioni cioè sollevate dalla "critica dei principi"; il secondo alla geometria creativa nel suo sviluppo autonomo". 


\section{ÁNGEL AUGUSTO PASQUALE Un análisis de la unidad por analogía a partir de los modelos geométricos}

corazón como los que cuentan con su análogo. El descubrimiento de la función parece realizarse en comparación con la geometría, a partir de la abstracción de lo material. En un trabajo anterior (Pasquale, 2016) sugerí que los términos geométricos son prioritariamente aplicables a las partes de los animales en tanto denotan funciones. El medio, que es principio del movimiento, nunca puede ser motor inmóvil del movimiento, sino que mueve al ser movido. De hecho, en el siguiente capítulo del MA (capítulo 10) el medio se emparenta con las actividades del deseo y del pneûma, presentes en el corazón (703a4-16). Por otro lado, el término geométrico "centro", que no se menciona pero se supone por lo dicho en el capítulo I del $M A$, tiene la función de ser principio inmóvil del movimiento: las articulaciones, afirma Aristóteles, actúan como si fueran un centro $(698 \text { a /8) })^{13}$. Sin embargo, en la argumentación que nos compete del capítulo 9, Aristóteles defiende un centro único del animal, el alma, capaz de permanecer inmóvil y producir movimientos desde ella.

\section{Consideraciones finales}

A esta altura considero pertinente recordar la discusión sobre el método de la zoología en el primer capítulo del De Partibus Animalium. La locomoción de los animales es una de las actividades de los animales que aunque comparten la denominación se diferencian significativamente según sus géneros, por lo que no se presentan de modo unitario en su forma: hay vuelo, nado, marcha y reptación (639a30-b6). La investigación debe dar cuenta de las causas de cada género (640al4-15): mientras que con algunas actividades caeríamos en repetición, porque son muy semejantes para los distintos géneros de los animales, no es el caso de la locomoción, en la que el estudio debe explicar las notables diferencias entre ellos. Con esto en mente, sin embargo, los pasajes que presenté tratan al movimiento de los

\footnotetext{
13 Luego de conceder que lo que primero mueve debe ser inmóvil y que esto es evidente en los animales puesto que es imposible que haya movimiento de una parte si otra no está en reposo, y que esta es la razón por la cual los animales tenemos articulaciones (698a7-17), el autor afirma que "[los animales] usan sus articulaciones como

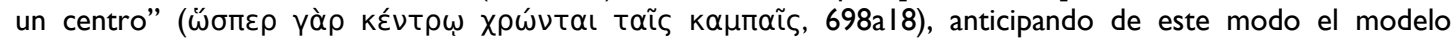
geométrico. El término geométrico "centro" aquí tiene la connotación de permanecer inmóvil incluso en el movimiento, por eso se le atribuye esta característica a la articulación, por la cual la parte que contiene la articulación puede pasar a ser una o dos, flexionarse o extenderse, cambiar potencial o actualmente (698al8-2I) El fin del diagrama es mostrar cómo la articulación se utiliza como un centro y que el movimiento se logra debido a la existencia de un punto de apoyo que está en la articulación. Una extremidad como la pierna puede moverse porque la rodilla tiene la capacidad de hacerse doble: la parte de la articulación próxima al muslo permanece fija mientras que la parte de la articulación próxima a la pierna se mueve con ella. La clave del movimiento por sí mismo de los animales es justamente la capacidad de doblarse en una parte que mueve y otra movida, capacidad que hasta el argumento del capítulo 9 tenían en cierta manera las articulaciones.
} 


\section{ÁNGEL AUGUSTO PASQUALE Un análisis de la unidad por analogía a partir de los modelos geométricos}

animales de una manera general, y la analogía es el recurso por el cual Aristóteles puede tratar la locomoción de los animales como semejante en cada caso mediante su función.

A través de este trabajo intenté mostrar que la analogía es la manera en que se relacionan los diferentes géneros del movimiento de los animales, y ésta muestra en primera medida lo que tienen de semejante. Sin embargo, el uso de la analogía en cada tratado sigue el propósito explícito de las investigaciones emprendidas. En el caso del $I A$ el recurso de la analogía resulta en la demarcación de las diferencias y una clasificación funcional de los animales según sus puntos de apoyo. En cambio, en el MA el recurso es empleado para dar con una explicación común del movimiento que desestime para este nivel de generalidad las diferencias entre los géneros comparados.

En ambos casos el recurso analógico actúa en un nivel doble. Por un lado, Aristóteles presenta modelos que pertenecen a otros dominios, relacionados con la geometría, que debían ser pretendidamente más familiares o conocidos, y así fundan el contexto heurístico de estos pasajes. Por su similitud en la función con los términos del foro -esto es, de la relación que se intenta dilucidar- nos ayudan a comprender algo de estos últimos. Por otro lado, la analogía supera el valor meramente heurístico y funda la pretendida unidad en el plano biológico mismo, en tanto es la manera en que se relacionan las facultades de los animales de diferentes géneros, y por tal motivo Aristóteles la emplea para explicar tanto sus diferencias como sus coincidencias.

\section{Referencias}

Bartolomé, S. \& Marcos, A. (2010). Aristóteles. Obra biológica (De Partibus Animalium, De Motu Animalium, De Incessu Animalium). Luarna.

Bénatouil, T. (2004). L'usage des anologies dans le De Motu Animalium. En A. Laks \& M. Rashed (eds.), Aristote et le mouvement des animaux. Dix études sur le De Motu Animalium (Pp. 8I-I I4). Presses Universitaires du Septentrion.

Candel, M. (1996). Aristóteles. Acerca del cielo. Meteorológicos. Gredos.

Carbone, A. (2005). La représentation de l'organisation spatiale du corps chez Aristote. Universite de Paris-Pantheon-Sorbonne.

De Groot, J. (2008). Dunamis and the Science of Mechanics: Aristotle on Animal Motion. Journal of the History of Philosophy, 46(I), 43-68.

Falcon, A. (2017). The Place of the De Motu Animalium in Aristotle's Natural Philosophy. En W. Wians \& R. Polansky (eds.), Reading Aristotle. Argument and Exposition (pp. 215-235). Brill. 
Jiménez Sánchez-Escariche, E. \& Alonso Miguel, A. (2000). Aristóteles. Partes de los animales. Marcha de los animales. Movimiento de los animales. Gredos.

Lennox, J. (2009). De caelo 2.2 and its debt to the De Incessu Animalium. En A.C. Bowen \& C. Wildberg (eds.), New perspectives on Aristotle's De caelo (pp. I87-2।4). Brill.

Lloyd, G.E.R. (1996). Unity of analogy. En Aristotelian Explorations (Pp. 138-159). Cambridge University Press.

Macagno, F. (2017). The logical and pragmatic structure of arguments from analogy. Logique \& Analyse, 240, 465-89.

Marcos, A. (1996). Aristóteles y otros animales: una lectura filosófica de la biología aristotélica. Promociones y Publicaciones Universitarias.

Nussbaum, M.C. (1985). Aristotle's De Motu Animalium. Princeton University Press.

Owen, G.E.L. (1986). Aristotelian Mechanics. En M. Nussbaum (ed.), Logic, Science, and Dialectics (pp. 315-333). Cornell University Press.

Pasquale, A.A. (2016). El modelo geométrico y el movimiento circular en el De Motu Animalium de Aristóteles. Tesis de grado. Universidad Nacional de La Plata. Facultad de Humanidades $y$ Ciencias de la Educación. http://www.memoria.fahce.unlp.edu.ar/tesis/te.1286/te.1286.pdf

Vita, V. (1982). II punto nella terminologia matematica greca. Archive for History of Exact Sciences, 27(2), I0I-114. 